\title{
Presence of benznidazole conjugated metabolites in urine identified by $\beta$-glucuronidase treatment
}

\author{
María Elena Marson ${ }^{\circledR 1,2,4, *}$, Facundo García Bournissen ${ }^{3,4}$, Jaime Altcheh ${ }^{3,4}$, Guillermo Moscatelli³, \\ Samantha Moroni ${ }^{3}$, Guido Enrique Mastrantonio ${ }^{1,2,4}$
}

\begin{abstract}
${ }^{1}$ Area de Toxicología, Departamento de Ciencias Biológicas, Facultad de Ciencias Exactas. Universidad Nacional de La Plata. La Plata, Provincia de Buenos Aires, Argentina, ${ }^{2}$ PlaPiMu-LaSeiSiC. Universidad Nacional de La Plata / Comisión de Investigaciones Cientificas de la Provincia de Buenos Aires, Argentina, ${ }^{3}$ Parasitología Chagas. Hospital de Niños Ricardo

Gutiérrez, Ciudad Autónoma de Buenos Aires, Argentina, ${ }^{4}$ Consejo Nacional de Investigaciones Científicas y Técnicas
\end{abstract}

(CONICET).

\begin{abstract}
Chagas disease is a serious public health problem in Latin America and, due to migration, in other nonendemic regions. Benznidazole (BNZ) is first choice drug in pediatric therapeutics. However, little is known regarding its metabolism in humans. The aim of the study was to isolate and identify products of human BZN metabolism in urine samples obtained from a pediatric Chagas patient and a healthy adult volunteer both treated with BZN. Urine samples were collected after dose of BNZ. Urine was treated with $\beta$-glucuronidase followed by an extraction procedure under two different $\mathrm{pH}$ conditions and a HPLC/UV and MS/MS identification of BZN and its metabolites. BZN (m/z 260.09847) was identified in all urine extracts. Peaks from each extracted chromatograms were selected for MS and MS/MS identification. Three compounds structurally related to BZN were identified: $\mathrm{BZN}-\mathrm{Na}^{+}(\mathrm{m} / \mathrm{z}$ 283.08009), N-amine-BZN (m/z 230.12307) and N-hydroxi-amine-BZN (m/z 246.11702). BNZ-Na ${ }^{+}$ was identified in all extracts, but $\mathrm{N}$-amine-BZN and $\mathrm{N}$-hydroxi-amine-BZN were only observed in those extracts treated with $\beta$-glucuronidase. This is the first experimental report showing elimination of BZN $\mathrm{N}$-reduced metabolites in urine. As they were released after treatment with $\beta$-glucuronidase it can be suggested that glucuronization plays a role in BNZ metabolism and renal elimination.
\end{abstract}

Keywords: Benznidazole metabolites. Chagas Disease/drug therapy. Glucuronidase/urine. High Performance Liquid Chromatography HPLC/methods. Mass Spectrometry/ methods. Antiparasitic agents/pharmacology.

\section{INTRODUCTION}

Chagas disease is one of the most neglected diseases in the world with approximately 10 million people infected in Latin America, where it is endemic. It is a zoonosis caused by infection with the parasite Trypanosoma cruzi (Schmunis, 2007; Urbina, Docampo, 2003). It has also expanded to non-endemic regions such as North American and European countries via migration of infected individuals (Schmunis, 1995WHO, 2002). For the past 40 years only two drugs, nifurtimox and benznidazole (BNZ), have been available for the treatment of Chagas disease, both with similar effectiveness and frequency of

\footnotetext{
*Correspondence: María Elena Marson. Campus Tecnológico CIC, Camino Centenario e/505 y 508 (1897), Gonnet, Provincia de Buenos Aires. Argentina.

Telephone: +54 221 5071291. E-mail: emarson@biol.unlp.edu.ar
}

adverse drug reactions (ADRs). However, they have long been used without a clear understanding of mechanisms of action, pharmacokinetics or toxicokinetics.

The mechanism of action of BZN ( $N$-benzyl-2nitroimidazole acetamide) is still not clear, but involves activation by a parasite nitroreductase (Hall, Wilkinson, 2012) to reactive metabolites that can induce reductive stress and covalent modification of proteins and other macromolecules in T. cruzi, disrupting parasite metabolism and function (Docampo, 1990; Moreno et al., 1982; Urbina, Docampo, 2003). To date, virtually nothing is known about the elimination pathways for BNZ in humans, it is assumed to be eliminated by the liver, with $<20 \%$ of the drug excreted unchanged by the kidney, however, virtually no information has been produced in humans (Raaflaub, Ziegler, 1979; Raaflaub, 1980; Lau et al., 1992), and little metabolism 
information is available from animal studies (Moreno et al., 1982; Richle, Raaflaub, 1982; Workman et al, 1984; Lee, Workman, 1986; Workman, Walton, Lee, 1986; Lee, Workman, Cheeseman, 1987; Walton, Workman, 1987).

The aim of this study was to isolate and identify products of human BZN metabolism in urine samples, including identification of conjugated products, by treating samples with $\beta$-glucuronidase. The experiments with $\beta$-glucuronidase and aril-sulfatase could give us the initial information about the presence of phase II BZN metabolites in the human urine samples. Both these enzymes converted the phase II BZN metabolites (conjugates of xenobiotics with glucuronic acid or sulfate) back to the phase I BZN metabolites.

\section{MATERIAL AND METHODS}

\section{Reagents}

Trichloroacetic acid (TCA) and dichloro methane (DCM) were purchased from Biopack (Buenos Aires, Argentina). Dimethyl sulfoxide (DMSO), anhydrous sodium sulfate, sodium dihydrogen phosphate dodecahydrate, potassium dihydrogenphosphate and sodium hydroxide were obtained from Anedra (Buenos Aires, Argentina). Hydrochloric acid and formic acid was obtained from J.T. Baker (USA). All reagents were analytic grade. Chromatographic grade demineralized water $(<0.2 \mu$ siemens $)$ was obtained in our laboratory with ionic exchange resins. For $\mathrm{pH}$ measures a $\mathrm{pH}$-meter from Altronix TPXII, and commercial $\mathrm{pH}$ test strips from Universal indicator Merck were used.

High-performance liquid chromatography HPLCgrade acetonitrile (J.T. Baker, USA) was used. Pure BNZ was obtained from Hoffmann-La Roche Ltd. (Buenos Aires, Argentina).

Helix pomatia $(H P) \beta$-glucuronidase (EC 3.2.1.31, type HP-2, 100,000 units $/ \mathrm{mL}$ ) with aril-sulfatase activity (EC 3.1.6.1, 7,500 units/mL) and a $\beta$-glucuronidase from Recombinant Escherichia coli BL21 (EC 3.2.1.31, 20,000,000 units/gr) (without aril-sulfatase activity) were obtained from Sigma Aldrich, USA. A phosphate buffer (pH 6.5) was prepared by mixing $800.0 \mathrm{~mL}$ of $0.067 \mathrm{M}$ sodium hydrogenphosphate dodecahydrate and $200.0 \mathrm{~mL}$ of $0.065 \mathrm{M}$ potassium dihydrogenphosphate and adjusted with hydrochloric acid.

\section{BZN Stock and Working Solutions}

BNZ stock solution was prepared with $112.8 \mathrm{mg}$ of BNZ in $5 \mathrm{~mL}$ of DMSO to complete dissolution and then accurately diluted with acetonitrile to $25.00 \mathrm{~mL}$ in a calibrated volumetric flask to obtain a $4.512 \mathrm{mg} / \mathrm{mL}$ solution. Stock solution was fractionated and stored at $4{ }^{\circ} \mathrm{C}$ and $-21^{\circ} \mathrm{C}$, and stability chromatographically evaluated from three to six months at both temperatures. Variable volumes of the BNZ stock solution were diluted in the mobile phase to obtain BZN working solutions.

\section{HPLC - UV/MS/MS instrumentation}

Urine samples were initially analyzed by HPLC/ $\mathrm{UV}$ for BNZ quantification at $313 \mathrm{~nm}$. Then, the extracts were analyzed by HPLC/UV for BZN and BZN metabolite peak identification at $220 \mathrm{~nm}$. Finally were analyzed HPLC/MS/MS for metabolite structure identification. The final determination about the structures of BZN phase metabolites was obtained by HPLC/MS/MS.

Quantification of BZN was done in both urine samples following a validated HPLC/UV method previously developed (Marson et al., 2013). The analysis for BZN and BZN metabolites peak identification was done with a HPLC Merck-Hitachi LC-6200A and MerckHitachi UV/Vis L-4250 detector, adjusted in $220 \mathrm{~nm}$. Separation was carried out at room temperature using a Lichrospher column $(5 \mu \mathrm{m}, 125 \mathrm{~mm}$ x $4.6 \mathrm{~mm}$ I.D. RP 18). Samples were injected with a $20 \mu \mathrm{L}$ sample loop. Mobile phase was Water/Acetonitrile (75:25) and flow was $1 \mathrm{~mL} / \mathrm{min}$.

For metabolite structure identification, water/ Acetonitrile urine extracts were collected and analyzed by using an Agilent liquid chromatograph series 1200 RRLC with a diode array detector (DAD) in tandem with a Bruker micrOTOF-QII electrospray source ionization (ESI) mass spectrometer. Samples $(5 \mu \mathrm{L})$ were separated on a Phenomenex Luna C18 $3 \mu \mathrm{m} \mathrm{100A} \mathrm{(100} \mathrm{x} 2 \mathrm{~mm})$, and were eluted in isocratic mode with a mobile phase of $70 \%$ water ( $0.1 \%$ formic acid): $30 \%$ acetonitrile at a flow rate of $0.2 \mathrm{~mL} / \mathrm{min}$. UV Detection wavelength was $220 \mathrm{~nm}$. Their $\mathrm{m} / \mathrm{z}$ values were determined by positive ESI. Positive ESI-tandem mass spectrometry (MS) was performed in automatic mode. MS was carried out with a drying gas temperature of $200^{\circ} \mathrm{C}$, a drying gas flow of 8.0 liters $/ \mathrm{min}$, a nebulizer gas pressure of $4 \mathrm{Bar}$, and a capillary voltage of $150.0 \mathrm{~V}$ in full-scan mode in the $\mathrm{m} / \mathrm{z}$ range of 50 to 900 .

\section{Urine samples and ethics statement}

A twenty-four hour urine sample was obtained from an 8 year old boy, $40 \mathrm{~kg}$ of weight, Chagas disease pediatric patient under treatment with BZN ( $5 \mathrm{mg} / \mathrm{kg} / \mathrm{d}$ bid p.o.) for 60 days (100 mg Radanil 
tablets, Roche, São Paulo, Brazil) (WHO, 2002; Altcheh et al., 2011) in the context of a pediatric clinical trial (Altcheh et al., 2014). The clinical protocol was approved by the Ethics and Research Review Committee, Buenos Aires Children's Hospital "Ricardo Gutierrez", and the Argentine National Drug and Food Administration (ANMAT), Ministry of Health, Argentina. Written informed consent was obtained from parent or legally authorized representatives as well as the assent from the pediatric patient. The study was registered in clinicaltrials. gov (\#NCT00699387). The sample was collected on day 15 after the start of treatment. The urine sample was stored at $-21^{\circ} \mathrm{C}$ until analysis. This patient, was randomly selected from a prospective pediatric population pharmacokinetic study of BNZ (Altcheh et al., 2014). The treatment of this patient was in agreement with current pediatric treatment guidelines (WHO, 2002).

A twenty-four hour urine sample was obtained from a healthy adult male, volunteer, (47 years old, 80 kilograms of weight) as a control for chromatographic parameters and BZN stability studies on urine matrix. The volunteer had not taken any medication for at least two weeks before sample collection. After providing the control sample, the same volunteer took one $400 \mathrm{mg}$ dose of BZN $(5 \mathrm{mg} / \mathrm{kg} / \mathrm{d}$ p.o. $)$ at night, and a 24 hour urine sample was collected. No pharmacodynamics or pharmacokinetics was proposed for this sample collection, it was taken, analogously as in the pediatric sample, in order to aisolate and identify products of $\beta$-glucuronidase reaction that could possibly be BZN metabolites. Written informed consent was also obtained from this volunteer.

\section{Urine sample pretreatment}

Stability of BZN in urine at $-21{ }^{\circ} \mathrm{C}$ was evaluated by chromatographic analysis (Marson et al., 2013). Four drug-free urine samples $(5.00 \mathrm{~mL}$ each) were spiked with BZN to attain a final concentration of $15 \mu \mathrm{g} / \mathrm{mL}$; a control sample without BNZ was kept under the same conditions. All samples were stored at $-21^{\circ} \mathrm{C}$, and thawed after 1,15 , 30 or 60 days, respectively. Every sample was treated after storage with $\beta$-glucuronidase and injected into the HPLC.
Enzymatic reactions: Three $5.00 \mathrm{~mL}$ aliquots were obtained in duplicates from the homogenized twentyfour hour urine samples (i.e. patient urine, volunteer control urine and volunteer exposed to BNZ urine). Aliquots (A) were treated with $E$. coli $\beta$-glucuronidase enzyme; Aliquots (B) were treated with $H$. Pomatia $\beta$-glucuronidase - arilsulfatase enzyme, and the aliquots (C) were left enzymatically untreated as controls. All three aliquots were processed together and under the same experimental conditions. Reaction temperature $\left(37^{\circ} \mathrm{C}\right)$, $\mathrm{pH}$ (6.5) amounts of enzyme and time of enzymatic reaction followed the optimal conditions prescribed by the supplier. Also, the amount of enzyme was calculated considering an excess of enzyme respect to the total amount of BZN dose. Temperature and $\mathrm{pH}$ were controlled and registered during reaction with a manual thermometer and a $\mathrm{pH}$-meter respectively. For aliquot $\mathrm{C}$, enzyme was replaced by an equal volume of buffer phosphate (Table I).

The enzymatic reaction was stopped by deproteinization with $500 \mu \mathrm{L}$ of TCA $30 \%(\mathrm{w} / \mathrm{v})$. Each aliquot was separated into two portions for extraction at two $\mathrm{pH}$ conditions: acidic ( $\mathrm{pH} 3)$ and basic $(\mathrm{pH} 9)$. Acidic conditions were obtained by addition of TCA $30 \%(\mathrm{w} / \mathrm{v})$ as described above, and basic conditions were obtained by adding $\mathrm{NaOH} 10 \%$ (w/v). Subsequent BZN extraction was done following a previously reported method for HPLC/UV detection (Marson et al., 2013) which consists in a liquid/liquid extraction of the urine samples with DCM and anhydrous sodium sulfate (near saturation), by shaking the mixture and centrifugation. The organic phases of three consecutive liquid/liquid extraction procedures were recovered together and evaporated to dryness (Table II). The residue was resuspended in $800 \mu \mathrm{L}$ of the HPLC/UV mobile phase and injected into the chromatographic system.

\section{RESULTS}

No significant BNZ concentration loss at $4{ }^{\circ} \mathrm{C}$ or $-21^{\circ} \mathrm{C}$ was observed in the reference stock solution and work solution after three and six months, respectively (relative standard deviation $\mathrm{RSD}<5 \%$ ). In addition,

TABLE I - Experimental conditions for a three hour enzymatic assay for $5 \mathrm{~mL}$ urine samples

\begin{tabular}{lccc}
\hline & Aliquot A & Aliquot B & Aliquot C \\
\hline Enzyme volume $(\mu \mathrm{L})$ & 10 & 10 & - \\
Buffer volume $(\mu \mathrm{L})$ & - & - & 10 \\
Temperature $\left({ }^{\circ} \mathrm{C}\right)$ range during reaction & $36.5-37.5$ & $36.5-37.5$ & $36.5-37.5$ \\
pH during reaction range & $6.52 \pm 0.25$ & $6.50 \pm 0.30$ & $6.53 \pm 0.15$ \\
\hline
\end{tabular}


TABLE II - Extraction procedure from urine samples after enzymatic treatment

\begin{tabular}{lcccccc}
\hline & $\begin{array}{c}\text { Urine Vol } \\
(\boldsymbol{\mu} \mathbf{L})\end{array}$ & $\begin{array}{c}\text { Vol }(\boldsymbol{\mu L}) \mathbf{N a O H} \\
(\mathbf{1 0 \%} \mathbf{p} / \mathbf{v})\end{array}$ & $\begin{array}{c}\mathbf{V o l}(\boldsymbol{\mu L}) \\
\mathbf{D C M} / \\
\mathbf{e x t r a c t i o n}\end{array}$ & $\begin{array}{c}\text { Total DCM } \\
\text { extraction Vol } \\
(\boldsymbol{\mu} \mathbf{L})\end{array}$ & $\begin{array}{c}\mathbf{N a}_{2}\left(\mathbf{S O}_{\mathbf{4}}\right)_{2} \\
(\mathbf{m g})\end{array}$ & Extract $\mathbf{p H}$ \\
\hline Aliquot A & 1200 & 30 & 400 & 1200 & 100 & 3 \\
& 1200 & 80 & 400 & 1200 & 100 & 10 \\
Aliquot B & 1200 & 30 & 400 & 1200 & 100 & 3 \\
& 1200 & 80 & 400 & 1200 & 100 & 10 \\
Aliquot C & 1200 & 35 & 400 & 1200 & 100 & 3 \\
& 1200 & 70 & 400 & 1200 & 100 & 8 \\
Urine blank control & 1200 & - & 400 & 1200 & 100 & 5 \\
& 1200 & 35 & 400 & 1200 & 100 & 12 \\
\hline
\end{tabular}

no decomposition products were observed in the chromatograms. Similarly, no significant BNZ loss was observed in spiked control urine samples after 1, 15, 30 and 60 days of storage at $-21^{\circ} \mathrm{C}(\mathrm{RSD}<5 \%)$. Also, no potential interference signal was observed in the drug-free control urine sample analyzed.

The BZN quantification results were $9.7 \pm 0.3 \mu \mathrm{g} / \mathrm{mL}$ and $25.2 \pm 0.2 \mu \mathrm{g} / \mathrm{mL}$ for adult and pediatric samples respectively, reflecting $\sim 3 \%$ and $\sim 7 \%$ of BNZ of the oral doses. BZN was identified in all urine extracts in HPLC/ UV chromatograms. Enzymatically treated extracts showed significantly different chromatographic profiles from non-treated ones. Figure 1 shows representative chromatograms obtained from the extracts analyzed, and those enzymatically treated generated extracts with greater amount of compounds identifiable by the chromatographic system used. Retention time for BZN in all extracts was $(8.0 \pm 0.1)$ minutes in HPLC/UV chromatograms.

Peaks (3 to 5) from each HPLC/UV chromatogram were selected for MS and MS/MS identification, by visual comparison between chromatogram profiles from those extracts enzymatically and non-enzymatically treated.

Retention time for BZN in all extracts was $(17.4 \pm 0.1)$ minutes in HPLC/MS/MS. Mass spectra of the selected chromatographic peaks were evaluated, and the principal molecular ions $\left[\mathrm{M}+\mathrm{H}^{+}\right]$analyzed for fragmentation patterns (+MS2) to confirm their structure. Three compounds structurally related to BZN were identified by HPLC/MS/MS: BZN-Na (m/z 283.08009), $N$-amine-BZN (m/z 230.12307) and $N$-hydroxy-amine$\mathrm{BZN}(\mathrm{m} / \mathrm{z} 246.11702)$. BZN-Na ${ }^{+}$is a technical artifact. This adduct, formed during extracts dilution with mobile phase, is differently resolved from the peak of free BNZ (Hall, Wilkinson, 2012). Pharmacologically, BNZ-Na ${ }^{+}$and $\mathrm{BNZ}$ should be considered as the same substance. BNZ $(\mathrm{m} / \mathrm{z} 260.09847)$ was identified in all extracts, but $\mathrm{N}$-amineBZN and $N$-hydroxy-amine-BZN were only observed in
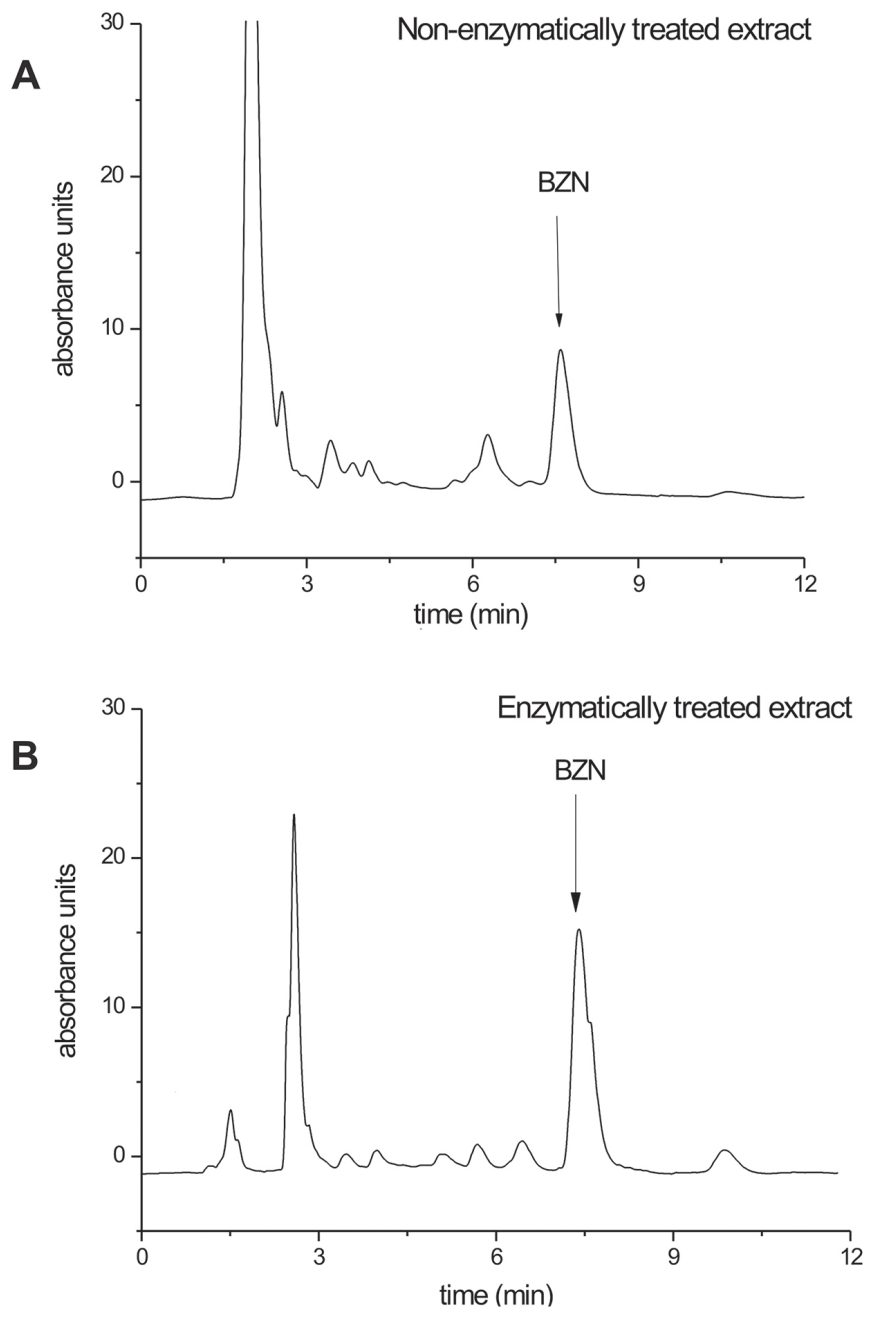

FIGURE 1 - Qualitative comparison of chromatographic profiles: A Example of a non-enzymatically treated urine extract chromatogram. B Example of an enzymatically treated urine extract chromatogram.

those extracts with enzymatic treatment, suggesting that they were excreted into urine in a conjugated form (i.e. glucuronated). No significant differences were found 
between extracts products from aliquots treated either with $\beta$-glucuronidase or arilsulfatase enzymatic activity, suggesting that the conjugated products are glucuronated and not sulfated. When analyzing the intensity of the signal at MS detection for the compounds identified, the extraction procedure at basic conditions showed higher results for $\mathrm{N}$-amine-BZN than for acidic extracts.

Table III shows $\mathrm{m} / \mathrm{z}$ of the compounds, molecular formula (MF), insaturation number (IN), + MS2 fragmentation pattern and the extract in which they were identified. The MS spectrum and MS/MS fragmentation profiles for every structure are shown in Figure 2. The new compounds identified had lower retention time $(10.7 \pm 0.2) \mathrm{min}$ for $\mathrm{N}$-amine-BZN, and $(11.2 \pm 0.1) \mathrm{min}$ for N-hydroxi-amine-BZN compared to BZN.

\section{DISCUSSION}

BNZ is the most commonly used drug in Latin America for the treatment of Chagas disease (Jannin, Villa, 2007). Trypanocidal activity was discovered empirically more than four decades ago. The drug, a nitroimidazole derivative, is believed to exert its biological activity through the enzymatic bioreduction of the nitro group with generation of reactive intermediates, a common mechanism of action of nitroheterocyclic compounds that generally act as pro-drugs and undergo activation to mediate their cytotoxic effects (Castro, Mecca, Bartel, 2006).

On the other hand, it has been suggested that BNZ serum concentrations do not appear to be related to the appearance of serious ADRs in adult patients with Chagas disease (Pinazo et al., 2013). This fact suggests a role of circulating metabolites in BNZ toxicity at therapeutic doses.
Unfortunately, little information is available on BNZ metabolism or about the identity of its main human metabolites, and apart from our previous work in which we identified the plasma metabolite $N$-benzylacetamide (Marson et al., 2015), no metabolite had previously been identified in human beings. This situation is significantly worse in the case of children.

In a murine model, enzymatic type II nitroreduction of $\mathrm{BZN}$ is proposed to result in the $\mathrm{BNZ}$ amino derivative (amine-BZN) through a series of sequential reactions with very low redox potential (Walton, Workman, 1986). Also, a type I nitroreduction pathway is proposed in which the latter derivative produces a highly toxic intermediate for the parasite and the host. In the latter case, parasite-specificity would be given by the parasiterestricted expression of type I nitroreductases (Hall, Wilkinson, 2012). A T. cruzi BNZ metabolomics study identified a large number of metabolites including the amine derivative, and hydroxylamine derivative (Trochine, Creek, 2014). Other researchers have proposed $\mathrm{N}$-oxide-molecules containing heterocycles as promising compounds with tripanocidal activity (Boiani et al, 2010).

Observations from chromatographic profiles showed a significant predominance of unchanged BZN compared with other substances derived from this parent drug. These compounds eluted before BZN, suggesting a more polar behavior according to the chromatographic system used. No differences in the profile of compounds identified were observed between the samples from the adult healthy volunteer and those from the pediatric patient. Also, the extraction procedure at different $\mathrm{pH}$ conditions did not show qualitatively different chromatographic profiles, except for the case of $N$-amine-BZN, where signal intensity found was around double from acidic ones. This suggests

TABLE III - HPLC/MS/MS identified compounds related to BZN, their m/z and molecular formula (MF), instauration number (IN), and the extract in which they were identified.

\begin{tabular}{|c|c|c|c|c|c|}
\hline $\mathbf{m} / \mathbf{z}$ & MF & IN & $\begin{array}{c}\text { +MS2 fragmentation pattern: } \\
\text { MF \& } \mathbf{m} / \mathbf{z}\end{array}$ & Structure Named & Extract presence \\
\hline 260.09847 & $\mathrm{C}_{12} \mathrm{H}_{12} \mathrm{~N}_{4} \mathrm{O}_{3}$ & 9 & $\begin{array}{c}\mathrm{C}_{12} \mathrm{H}_{12} \mathrm{~N}_{3} \mathrm{O}(214.09749) \\
\mathrm{C}_{9} \mathrm{H}_{10} \mathrm{NO}(148.07569) \\
\mathrm{C}_{7} \mathrm{H}_{7} \mathrm{O}(107.04914) \\
\mathrm{C}_{7} \mathrm{H}_{7}(91.05423)\end{array}$ & $\mathrm{BZN}$ & In all extracts \\
\hline 283.08009 & $\mathrm{C}_{12} \mathrm{H}_{12} \mathrm{~N}_{4} \mathrm{O}_{3} \mathrm{Na}$ & 9 & Same as above & $\mathrm{BZN} \mathrm{Na}^{+}$ & In all extracts \\
\hline 230.12307 & $\mathrm{C}_{12} \mathrm{H}_{14} \mathrm{~N}_{4} \mathrm{O}$ & 8 & $\mathrm{C}_{5} \mathrm{H}_{6} \mathrm{~N}_{3} \mathrm{O}(124.05054)$ & $\mathrm{NH}_{2}-\mathrm{BZN}$ & $\begin{array}{l}\text { Only in enzymatically } \\
\text { treated extracts }\end{array}$ \\
\hline 246.11702 & $\mathrm{C}_{12} \mathrm{H}_{14} \mathrm{~N}_{4} \mathrm{O}_{2}$ & 8 & $\begin{array}{c}\mathrm{C}_{5} \mathrm{H}_{6} \mathrm{~N}_{3} \mathrm{O}_{2}(140.04545) \\
\mathrm{C}_{4} \mathrm{H}_{6} \mathrm{~N}_{3} \mathrm{O}(112.05054) \\
\mathrm{C}_{7} \mathrm{H}_{7}(91.05423) \\
\end{array}$ & NH-OH-BZN & $\begin{array}{l}\text { Only in enzymatically } \\
\text { treated extracts }\end{array}$ \\
\hline
\end{tabular}



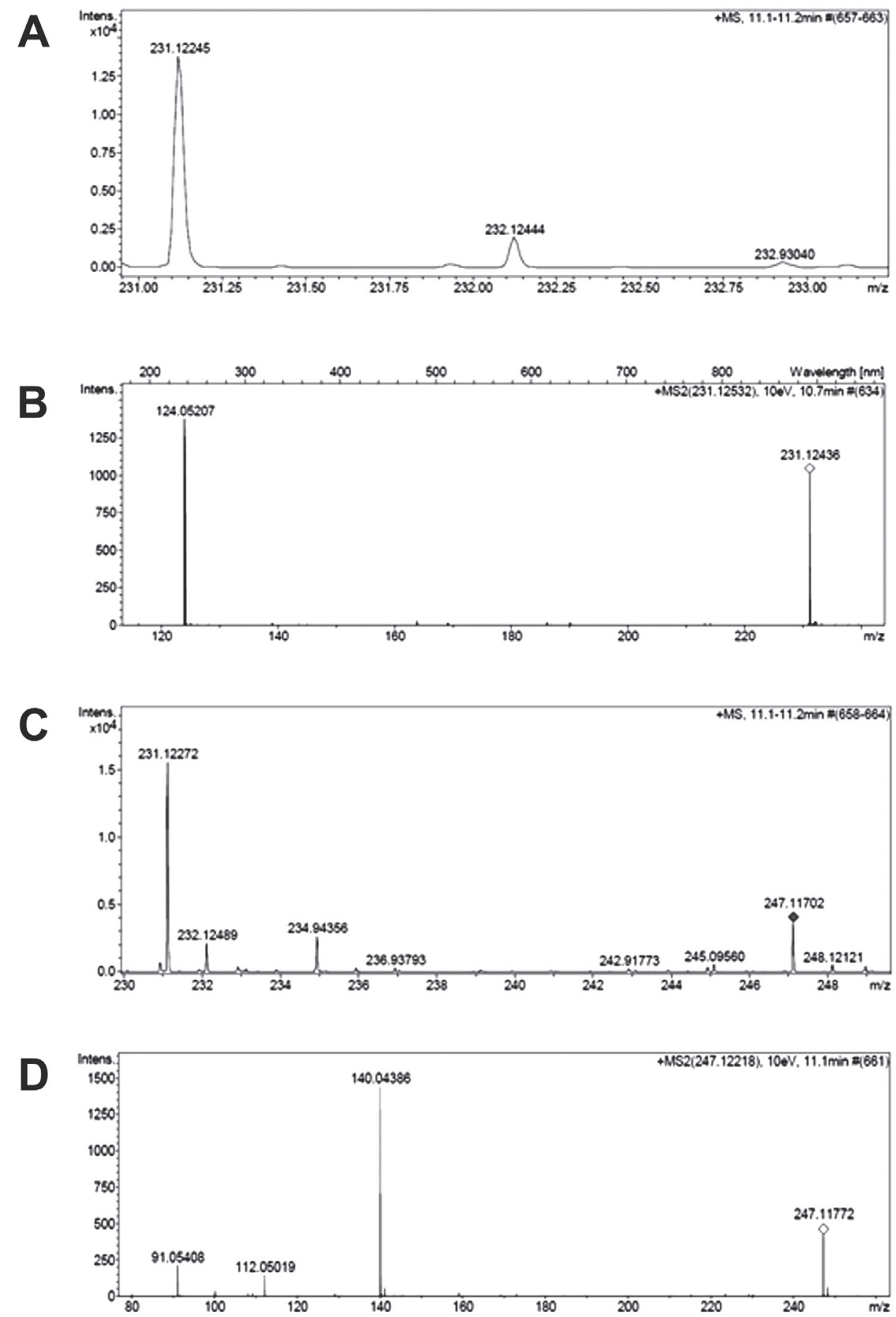

FIGURE 2 - A Amine-BNZ(H) ${ }^{+}$MS detection (m/z 231.12245; Retention time 10.7 minutes), scanning 50 to $800 \mathrm{~m} / \mathrm{z}$; B +MS2 for $\mathrm{m} / \mathrm{z} 231.12532$ and Retention time of 10.7 minutes; $\mathbf{C}$ N-hydroxi-amine-BZN(H) $)^{+}$MS detection $(\mathrm{m} / \mathrm{z} 247.11702$; Retention time 11.1-11.2 min), scanning 50 to $800 \mathrm{~m} / \mathrm{z}$; D $+\mathrm{MS} 2$ for $\mathrm{m} / \mathrm{z} 247.12218$ and Retention time of 11.1 minutes.

that for this compound it would be better to select basic conditions for the extraction process. As we did not have these compounds synthesized in conditions so as to be used as an analytical standard, accurate quantification was not done. In general terms, these compounds could be extracted either in acidic or basic conditions, but we recommend a basic extraction for better recovery of $\mathrm{N}$-amine-BZN .
Based on the mass spectrometry detection and identification results, we conclude that the amine-BZN and N-hydroxiamine-BZN could be phase I metabolic products of BNZ, excreted in urine as conjugates (as they only were present in glucuronidase-treated extracts). As none of the metabolites were identified in the enzymatically untreated extracts it is possible to conclude that these molecules were eliminated 


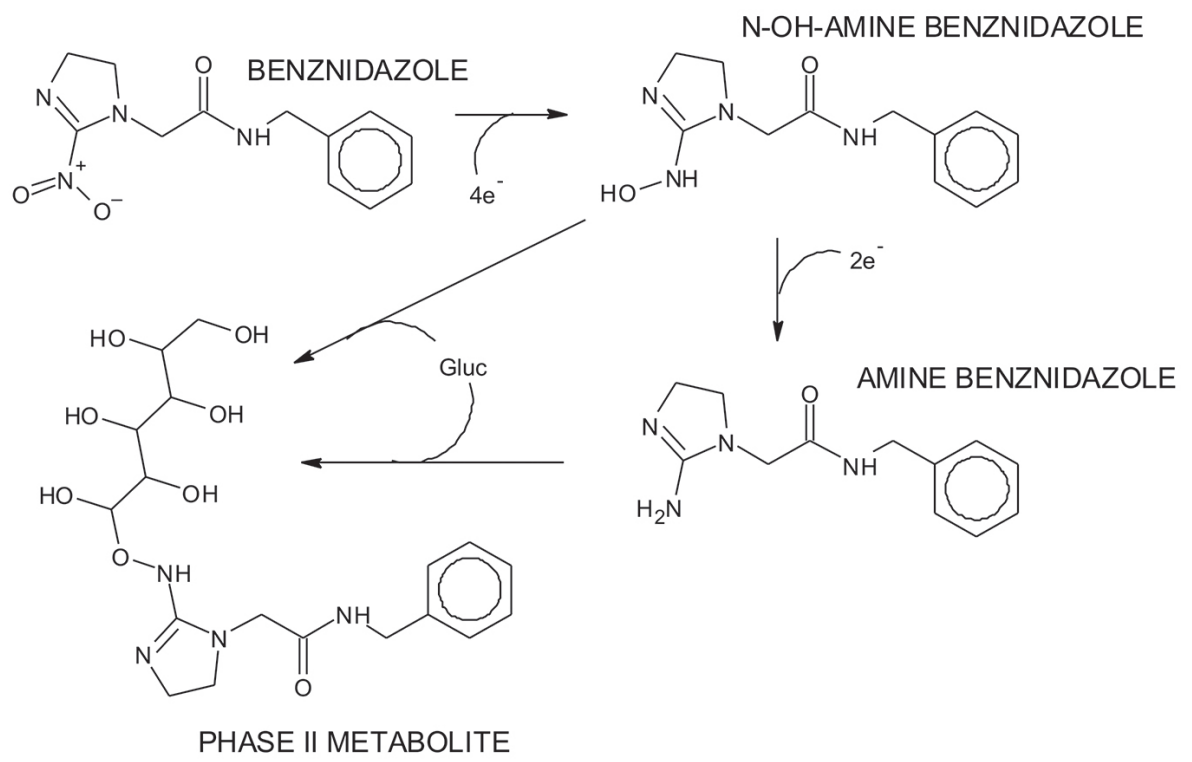

FIGURE 3 - Proposed metabolic pathway of BZN and its metabolites production.

in their $N$-glucuronides forms. Although these results are limited by the small number of samples studied, they offer interesting new hypotheses that need to be confirmed. We present in Figure 3 a hypothetic metabolic pathway from $\mathrm{BZN}$ to these compounds.

\section{CONCLUSION}

In this work, for the first time, two BZN metabolites ( $N$-amine-BZN and $N$-hydoxy-amine-BZN) are identified in human urine samples. These metabolites are likely phase I metabolites, product of xenobiotics metabolic reactions that convert the BNZ nitro group into the amine or $N$-hydroxy-amine group, respectively. $N$-glucuronides conjugates are an example of phase II reaction products that increase the polarity of the molecules to facilitate excretion into urine. This work provides experimental evidence of benznidazole $N$-reduced metabolites and its renal excretion in $\mathrm{N}$-glucuronide form.

\section{REFERENCES}

Altcheh J, Moscatelli G, Moroni S, Garcia-Bournissen F, Freilij $H$. Adverse events after the use of benznidazole in infants and children with Chagas disease. Pediatrics. 2011;127(1):212-8. doi:10.1542/peds.2010-1172.

Altcheh J, Moscatelli G, Mastrantonio G, Moroni S, Giglio N, Marson ME et al. Population Pharmacokinetic Study of Benznidazole in pediatric Chagas disease suggests efficacy despite lower plasma concentrations than in adults. PLoS Negl Trop Dis. 2014;8(5):e2907. doi:10.1371/journal.pntd.0002907.
Boiani M, Piacenza L, Hernández P, Boiani L, Cerecetto H, González M, Denicola A. Mode of action of Nifurtimox and $\mathrm{N}$-oxide-containing heterocycles against Trypanosoma cruzi: Is oxidative stress involved? Biochem Pharmacol. 2010;79(12):1736-45. doi: 10.1016/j.bcp.2010.02.009.

Castro JA, Mecca MM, Bartel LC. Toxic side effects of drugs used to treat Chagas' disease (American trypanosomiasis). Hum Exp Toxicol. 2006;25(8):471-9.

Docampo R. Sensitivity of parasites to free radical damage by antiparasitic drugs. Chem Biol Interact. 1990;73(1):1-27.

Hall BS, Wilkinson SR. Activation of BNZ by Trypanosomal Type I Nitroreductases results in glyoxal formation. Antim Agents Chemother. 2012;56(1):115-23. doi: 10.1128/ AAC.05135-11.

Jannin J, Villa L. An over view of Chagas disease treatment. Mem Inst Oswaldo Cruz. 2007;102(Suppl 1):95-7.

Lau AH, Lam NP, Piscitelli SC, Wilkes L, Danziger LH. Clinical pharmacokinetics of metronidazole and other nitroimidazole anti-infectives. Clin Pharmacokinet.1992;23(5):328-64.

Lee FY, Workman P. Altered pharmacokinetics in the mechanism of chemosensitization: effects of nitroimidazoles and other chemical modifiers on pharmacokinetics, antitumour activity and acute toxicity of selected nitrogen mustards. Cancer Chemother Pharmacol.1986;17(1):30-7. 
Lee FY, Workman P, Cheeseman KH. Misonidazole and benznidazole inhibit hydroxylation of CCNU by mouse liver microsomal cytochrome P-450 in vitro. Biochem Pharmacol. 1987;36(8):1349-55.

Marson ME, Dana, DD, Altcheh J, García Bournissen F, Mastrantonio G. Development of UV/HPLC methods for quantitative analysis of benznidazole in human plasma and urine for application in pediatric clinical studies. J Clin Lab Anal . 2013;27(5),384-90.

Marson ME, Altcheh J, Moscatelli G, Moroni S, GarcíaBournissen F, Mastrantonio GE. Identification of $\mathrm{N}$-benzylacetamide as a major component of human plasmatic metabolic profiling of benznidazole. Eur J Drug Metab Pharmacok. 2015;40(2),209-17. doi: 10.1007/s13318-0140195-8.

Moreno SN, Docampo R, Mason RP, Leon W, Stoppani AOM. Different behaviors of benznidazole as free radical generator with mammalian and Trypanosoma cruzi microsomal preparations. Arch Biochem Biophys. 1982;218(2):585-91.

Pinazo MJ, Guerrero L, Posada E, Rodríguez E, Soy D, Gascon J. Benznidazole-related adverse drug reactions and their relationship to serum drug concentrations in patients with chronic Chagas disease. Antimicrob Agents Chemother. 2013;57(1):390-5.

Raaflaub J, Ziegler WH. Single-dose pharmacokinetics of the trypanosomicide undeter-benznidazole in man. Arzneimittelforschung. 1979;29 (10):1611-4.

Raaflaub J. Multiple-dose kinetics of the trypanosomicide benznidazole in man. Arzneimittelforschung.1980;30(12):2192-4.

Richle RW, Raaflaub J. Difference of effective antitrypanosomal dosages of benznidazole in mice and man: chemotherapeutic and pharmacokinetic results. Acta Trop. 1982;37(3):257-61.
Schmunis G. Risk of Chagas disease through transfusions in the Americans. Clin Infect Dis. 1995;21(3):551-5.

Schmunis G. Epidemiology of Chagas disease in non-endemic countries: the role of international migration. Mem Inst Oswaldo Cruz. 2007;102(Suppl 1):75-85.

Trochine A, Creek DJ. Benznidazole biotransformation and multiple targets in Trypanosoma cruzi revealed by metabolomics. PLoS Negl Trop Dis. 2014;22;8(5e2844):1-15. doi: 10.1371/journal.pntd.0002844.

Urbina JA, Docampo R. Specific chemotherapy of Chagas disease: controversies and advances. Trends Parasitol 2003;19(11):495-501.

Walton MI, Workman P. Reversed-phase high performance liquid chromatographic method for the simultaneous determination of the 2-nitroimidazole-BNZ and its amine metabolite in biological fluids. J Chromat. 1986;375:190-6.

Walton MI, Workman P. Nitroimidazole bioreductive metabolism: quantitation and characterisation of mouse tissue benznidazole nitroreductases in vivo and in vitro. Biochem Pharmacol. 1987;36(6):887-96.

WHO Expert Committee on the Control of Chagas disease: second report of the WHO Expert Committee. 905.ed. Geneva: World Health Organization; 2002.

Workman P, White RA, Walton MI, Owen LN, Twentyman PR. Preclinical pharmacokinetics of benznidazole. Br J Cancer. 1984;50(3):291-303.

Workman P, Walton MI, Lee FY. Benznidazole: nitroreduction and inhibition of cytochrome P-450 in chemosensitization of tumour response to cytotoxic drugs. Biochem Pharmacol. 1986;35(1):117-9.

Received for publication on $17^{\text {th }}$ January 2018 Accepted for publication on $08^{\text {th }}$ May 2018 